\title{
Peertechz
}

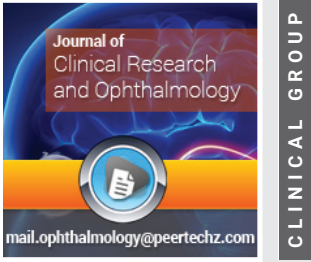

\section{Draw attention to the teratogenic effect of DMD in MS treatment}

\author{
Shi-Fang Hou', Yin Jian', Li-Yan Qiao' ${ }^{2}$ Zun-Bao Li ${ }^{3}$ Kai \\ Feng ${ }^{4}$ and $\mathrm{Xian}-\mathrm{Hao} \mathrm{Xu}^{1 *}$ \\ ${ }^{1}$ Department of Neurology, Beijing Hospital, National Center of Gerontology; Institute of Geriatric \\ Medicine, Chinese Academy of Medical Sciences, China \\ ${ }^{2}$ Department of Neurology, Yu Qua Hospital, Qing Hua University, Beijing, China \\ ${ }^{3}$ Department of Neurology, Gao Xin Hospital, Xian, China \\ ${ }^{4}$ Department of Neurology, Shunyi Hospital, Capital Medical University, Beijing, China
}

Received: 05 September, 2020
Accepted: 17 September, 2020

Published: 18 September, 2020

*Corresponding authors: Xian-Hao XU, Department of Neurology, Beijing Hospital, National Center of Gerontology; Institute of Geriatric Medicine, Chinese Academy of Medical Sciences, China,

E-mail: xuxianhao99@163.com

https://www.peertechz.com

Check for updates
More than 2.3 million people are affected by Multiple Sclerosis (MS) worldwide. Since the 1990s, disease modifying drug (DMD) has been developing increasingly. With more and more clinical applications, more and more attention has been paid to the adverse effects of DMD in the treatment of MS. DMD selection for patients with MS during pregnancy is one of the most important topics for neuroimmunologists and obstetrics. This comment is mainly based on our published and forthcoming data.

\section{Multiple sclerosis}

MS is an autoimmune inflammatory demyelinating neurodegenerative disorder of Central Nervous System (CNS), clinically characterized by symptoms including blurred and/ or double vision, fatigue, impaired motor activity, bladder and bowel dysfunction, and cognitive impairment etc.

MS is usually developed in individuals with given genetic background and encountering special environment, such as infection especially EBV infection [1,2].

MS is an autoimmune disease, caused by dysfunction of immunologic system, it may associate with other autoimmune disease, such as myasthenia gravis etc [3]. A lot of hot spots and challenges had been studying [4-13].

The diagnosis is mainly based on dissemination in time and dissemination in space of lesions in CNS, and ruled out other diseases [4-16].
More than 2.3 million people are affected by MS worldwide. The prevalence of MS in Shanghai, China is 1.39 (F 1.80, M $0.98) \times 10^{-6}[17]$.

The $2.8 \%$ initial clinical manifestation of MS is ophthalmic (visual impairment and/or diplopia), most of them are first visited even hospitalized in department of ophthalmology [1].

During June 12 2011-July 31 2020, the number of hospitalized patients with MS was 69.

Oligoclonal bands (OCB) contributes to the diagnosis of MS [15-19]. Recently, it has been considered to be the evidence of dissemination in time of the lesion in CNS [17]. The number of patients who had OCB tests was 59. Four cases were abnormal.

The number of cases with extraocular muscle involvement (eye movement disorder, diplopia, blepharoptosis) was 5 . The number of cases with initial extraocular muscle involvement was 3.

The number of patients with optic nerve involvement (vision disturbance, pupil) was 25.

The number of patients with initial optic nerve involvement was 19.

The number of patients who had VEP examined was 59. The number of patients with prolonged $\mathrm{P}-100$ incubation period was 43. 
The number of patients who had BAEP inspected was 59 . The number of patients with abnormal BAEP was 10. The number of patients with 3-5 wave elongation was 9 .

The number of patients who had SSEP examined was 59. The number of patients with abnormal SSEP was 26 . The number of patients whose lesions located in cervical enlargement and above was 18 . The number of patients whose lesions located between cervical and lumbar enlargement was 6 . The number of patients whose lesions located in lumbar enlargement and below was 5 .

The number of patients who had MRI examined was 69. The number of patients with abnormal MRI was 62 . The number of patients whose lesions located in cortical and juxtacortex was 18. The number of patients whose lesions located in periventricular was 19. The number of patients whose lesions located in infratentorial was 10. The number of patients whose lesions located in spinal cord was 3.

\section{Incurable but treatable $[20,21]$.}

Although there is no cure for MS, effective strategies are available to treat exacerbations (relapses), modify the disease course, enhance their comfort, improve symptoms and quality of life.

\section{Treatment of MS}

\section{DMDs for MS therapy [20-22].}

DMDs have been shown to be efficacious in reducing relapse frequency in MS, have been shown to have a decreased risk of relapse, fewer emergency room visits, fewer severe relapses, fewer hospitalizations, fewer neuropsychological issues, lower costs, and increased quality of life.

DMDs included Interferon $\beta[22,23]$, hematopoietic stem cell transplantation [24, MMF [25], tacrolimus, glatiramer acetate [17], etc.

There has been no report of teratogenic effect of DMD in china. (http://www.cnki.net/).

Teratogenic effect of drug is very difficult to investigate, therefore there is rare report of teratogenic effect of drugs treating MS. Not have being discovered does not mean not exist. Even some is lethal. Call for paying attention to the teratogenic effect of DMD for MS is very important.

Draw special attention to our knowledge, no existing studies in the published literature have described the relationship between the use of glatiramer acetate and congenital diaphragmatic agenesis.

\section{References}

1. Xian-hao X (2000) editor-in-chief. Neuroimmunology. Wuhan: Hubei Science and Technology Press.

2. Barcellos LF, Thomson G, Carrington M, Schafer J, Begovich AB, et al. (1997) Chromosome 19 Single-Locus and Multi locus Haplotype Associations With
Multiple Sclerosis. Evidence of a New Susceptibility Locus in Caucasian and Chinese Patients. JAMA. 278: 1256-1261. Link: https://bit.ly/3mw8yxV

3. He JH, Zhang Z, Liu F, Xu XH (1998) A case report of myasthenia gravis coexisting with multiple sclerosis. Chinese Journal of Neurology 31: 50.

4. Xian-Hao X (1999) Chinese Journal of Neurology 32: 325-328.

5. Xian-Hao X (1999) Recent advances in neurology in China. Chinese Medical Journal 38: 615-618.

6. Xian-Hao X (2004) Advances in multiple sclerosis research (monograph) Chinese Journal of Neurology 37: 3-6.

7. Xian-Hao X (2005) Research progress of neuroimmunology in China. Chinese Journal of Neurology 38: 1-4.

8. Xian-Hao X (2007) Research progress of inflammatory demyelinating disease of nervous system. Chinese Journal of Neurology 40: 139-141.

9. Shi-Fang H, Xian-Hao X (2013) Summary of 2012 Pan Beijing Expert Forum on Multiple sclerosis, Treatment and Research Collaboration. Chinese Journal of Neuroimmunology and Neurology 20: 150-151.

10. Xian-Hao XU, Feng K, Zhang XH (2013) The developmental history of study on neuromyelitis optica. Chinese Journal of Neuroimmunology and Neurology 20: 77-78.

11. Hou SF, Liu YH, Xu XH (2014) Progress in diagnosis and treatment of multiple sclerosis. Chinese Journal of Modern Neurological Diseases 14: 849-853.

12. Qiao L, Xu XH, Hou SF (2014) Peripheral blood interleukin 4, 10 patients with multiple sclerosis and secretion of interferon - clinical research. Chinese Journal of Modern Neurological Diseases 14: 867-872.

13. Xu XH (2017) The past, present and future of neuroimmunology in China Chinese $\mathrm{J}$ Neuroimmunology and Neurology 24: 78-80.

14. Xu XH (2000) Advances in multiple sclerosis research. Chinese Journal of Neuroimmunology and Neurology 7: 133-134.

15. Xian-hao X, Jianjun $L$ (2007) The research hotspot and trend of multiple sclerosis. Chinese Journal of Medicine 87: 2737-2738.

16. Zhang X, Xu X (2007) Diagnosis of multiple sclerosis and optic neuromyelitis. Chinese Journal of Medical Sciences 87: 1739-1740.

17. Liu X, Guang Z (2019) editor-in-chief. Multiple Sclerosis $2^{\text {nd }}$ Edition. Peking University Medical Press, Beijing.

18. Xu X, McFarlin DE (1984) Oligoclonal bands in CSF: Twins with MS. Neurology 34: 769-774. Link: https://bit.ly/2RzkyR9

19. XU XH (1985) Detection of IgG Fraction Bands in Cerebrospinal fluid by Highly Sensitive and Specific Immunoperoxidase Staining Methods. Asian Pacific J Allergy Immunol 3: 9-15. Link: https://bit.ly/2RDhCCU

20. Xu X (2009) Drug therapy for relapsing-remitting multiple sclerosis. Chinese Journal of Neurology 42: 6-7.

21. Feng $\mathrm{K}, \mathrm{Xu} X$ (2013) An oral drug used to treat multiple sclerosis. Chinese Journal of Neuroimmunology and Neurology 20: 89-91.

22. Liu J, Yin J, Ye J, Sun W, Li C, et al. (2010) Effects of methylprednisolone on PERIPHERAL blood IL-12 and CXCL13 in patients with multiple sclerosis Journal of Brain and Neurological Diseases 18: 146-148.

23. Li ZX, Lu CZ, Zhang XH, Cui LY, Xu XH, et al. (2014) Interferon beta-1b is effective and has a favourable safety profile in Chinese patients with relapsing forms of multiple sclerosis. Neurology Asia19: 179-189. Link: https://bit.ly/2FBZyqB

24. Wang XQ, Qi XK, Xu XH (2000) Hematopoietic stem cell transplantation for multiple sclerosis. Chinese Journal of Neurology 33: 186-187.

25. Chen Z, Liu L, Peng D, Sun C, Xu X (2009) Clinical application of Mycophenol ester in autoimmune diseases. Chinese Journal of Neuroimmunology and Neurology 16: 144-147.

Copyright: @ 2020 Hou SF, et al. This is an open-access article distributed under the terms of the Creative Commons Attribution License, which permits unrestricted use distribution, and reproduction in any medium, provided the original author and source are credited. 